\title{
Preface to Volume II
}

Two volumes of the electrochemistry series in Topics in Current Chemistry are dedicated to new applications of electrochemical techniques. The contributions in Volume II cover two fields:

- Application of electrochemical methods as analytical tools for the detection as well as the concentration and activity determination of biologically active compounds in bioanalysis and medicine;

- Electrochemical processes for photoelectrochemical energy conversion using semiconductor electrodes or microparticles.

In the first two contributions electroanalytical techniques are described for application in bioanalysis and medicine. The increasing interest in this field is mainly due to the excellent selectivities and detection limits. In addition, the possibilities of miniaturization allow the development of in vivo analysis.

The following two papers deal mainly with problems in energy conversion, in particular, the transformation of irradiation energy into electrical or chemical energy. The present status and future possible developments of photoelectrochemical energy conversion is presented. In a second paper electrochemical developments are connected to colloidal chemistry and the application of colloidal particles as catalysts for electron transfer reactions and as photocatalysts are discussed.

These articles may show that electrochemical developments and studies not only influence a restricted area but are also important for a number of other fields.

Bonn, June 1987

Eberhard Steckhan 
